\title{
Post-buckling behaviour of a slender beam in a circular tube, under axial load
}

\author{
M. Gh. Munteanu ${ }^{1} \&$ A. Barraco ${ }^{2}$ \\ ${ }^{I}$ Transilvania University of Brasov, Romania \\ ${ }^{2}$ Ecole Nationale Supérieure d'Arts et Métiers ENSAM), Paris, France
}

\begin{abstract}
This paper deals with the study of the behaviour of a slender beam introduced in a cylindrical tube and subjected to an axial compressive force. The beam is very long compared to its transversal dimensions and therefore it will buckle to a very small axial force. The post-buckling behaviour is examined. The study has important applications in the petroleum industry, for coiled tubing in the case of drilling in horizontal or inclined wellbores. The slender beam has a constant cross-section that can have any form, although a circular cross-section is the most used in practice. The problem has a geometrical non-linearity to which the non-linearity caused by the friction has to be added. Rotations could be large and a special isoparametric 3D beam finite element is elaborated: the EulerRodrigues quaternion was preferred to describe the finite cross-section rotations. The paper presents only the static case, but extending the presented approach to dynamic analysis is quite natural. The method is very accurate and it is rapidly convergent due to the fact that the exact equations, written for the deformed configuration, are solved. The iterative Newton-Raphson method was used to solve the nonlinear differential equations.

Keywords: coiled tubing, finite element method, post-buckling behaviour, Euler quaternion, geometrical non-linearity.
\end{abstract}

\section{Introduction}

A long slender initially straight beam compressed and constrained within a circular cylinder is studied in this work. This problem presents a great interest in rock engineering and petroleum production. The beam buckles within the narrow space of a drill hole under the action of axial force and its own weight. Due to its 
length, the beam will first buckle according to the classic Euler formula, a sinusoidal buckling, when the displacements are considered infinitely small. The post-buckling behaviour is much more important because the beam lies on the cylinder and friction will occur that diminishes the axial load transmission. The problem is highly complicated because it involves a geometrical non-linearity to which the friction has to be added. Several theoretical models were proposed, all of them based on analytical models, some of them complicated, [1,2], or based on simplified (or even very simple) models using in general a variational approach, [3-12]. Experimental measurements were performed as well: some works are based only on experiments, [13, 14], while in other works the experiments were meant to verify the theoretical models, [1, 2, 4, 11, 12]. Almost all theoretical models assume that post-buckling shape is a helix that is not always true. Some of them consider even a constant pitch helix that it is obviously not exact mainly when the friction is taken into consideration, $[4,12]$.

In this work a numerical method based on finite element method is proposed. A special type of finite element is presented, based on some previous works, [15-17]. This finite element type is specially conceived for geometrical nonlinear beam systems and uses Euler-Rodrigues quaternion and the axial strain as generalized coordinates. It allows a very exact and fast convergent numerical model to be elaborated. The beam is initially straight and lies along a generatrix; it lies always on the cylindrical surface even after deformation due to the axial force. Two boundary conditions were examined, clamped-free beam and double hinged beam; significantly different results were obtained for these two cases.

\section{Beam theory}

Because of beam length, the Euler-Bernoulli beam theory is applied. The beam is considered initially straight and it has a constant cross-section. The first variation of the total potential energy has the expression:

$$
\delta \Pi=\delta U+\delta V=\underbrace{E A \int_{l} \delta \varepsilon_{0}{ }^{\mathrm{T}} \varepsilon_{0} \mathrm{~d} s+\int_{l} \delta \boldsymbol{\kappa}^{\mathrm{T}} \mathbf{D} \boldsymbol{\kappa} \mathrm{d} s}_{\delta U}-\underbrace{\left(\int_{l} \delta \boldsymbol{u}^{\mathrm{T}} \boldsymbol{p} \mathrm{d} s+\int_{l} \delta \boldsymbol{\varphi} \boldsymbol{m} \mathrm{d} s\right)}_{\delta V}=0 .
$$

where $U$ is the deformation energy, $V$ is the potential of the external load, $\boldsymbol{u}$ is the displacement vector, $\varphi$ represents the small rotation vector, $\boldsymbol{p}$ and $\boldsymbol{m}$ are the distributed force vector and the distributed moment vector, respectively, acting on the beam. Efforts and strains are linked by the relations (Hooke's low):

$$
N=E A \varepsilon_{0}, \quad \boldsymbol{M}=\mathbf{D} \boldsymbol{\kappa},
$$

where $N$ is the axial effort, $E$ is the Young modulus, $A$ the cross-section area and $\varepsilon_{0}$ is the axial deformation of the beam centreline; the moment vector $\boldsymbol{M}$ and the curvature $\kappa$ have following expressions in the local reference frame:

$$
M=\left(\begin{array}{lll}
M_{1} & M_{2} & M_{3}
\end{array}\right)^{T}, \quad \kappa=\left(\begin{array}{lll}
\kappa_{1} & \kappa_{2} & \kappa_{3}
\end{array}\right)
$$


$M_{\mathrm{t}}$ is the torque and $M_{2}, M_{3}$ are the bending moments; $\kappa_{1}, \kappa_{2}$ and $\kappa_{3}$ are the torsion and the two curvatures, that is the variation of angles between two local reference frames situated at the infinitesimal distance $d s$.The Hooke's low matrix $\mathbf{D}$ is

$$
\boldsymbol{M}=\mathbf{D} \boldsymbol{\kappa} \text { or }\left\{\begin{array}{l}
M_{t} \\
M_{2} \\
M_{3}
\end{array}\right\}\left[\begin{array}{ccc}
G I_{\mathrm{t}} & 0 & 0 \\
0 & E I_{2} & 0 \\
0 & 0 & E I_{3}
\end{array}\right]\left\{\begin{array}{l}
\kappa_{1} \\
\kappa_{2} \\
\kappa_{3}
\end{array}\right\} .
$$

$I_{\mathrm{t}}, I_{2}$ and $I_{3}$ are second order geometrical properties of the cross-section $\left(I_{2}\right.$ and $I_{3}$ are the principal inertia moments); $G$ is the transversal elasticity modulus.

The main difficulty in 3D geometrical non-linear study, in classical incremental formulation, is that the rotations form no longer a vector. This is why in this work the Euler-Rodrigues parameters are used to describe crosssection rotation, [15-17]. They are defined for a finite rotation of angle $\theta$ around the axis defined by the unit vector $v=\left(\begin{array}{lll}v_{x} & v_{y} & v_{z}\end{array}\right)^{T}$. The rotation is represented by the scalar $l_{0}=\cos \frac{\theta}{2}$ and by the vector $\sin \frac{\theta}{2} v$ grouped in the following column matrix, the well-known Euler-Rodrigues quaternion:

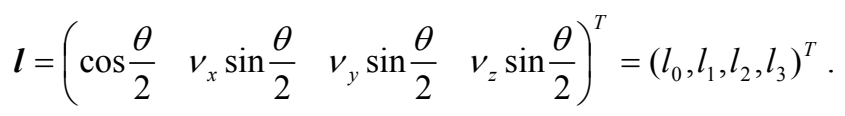

In this work the finite rotation $\theta$ transforms the initial infinite small length element $d s$ of the initial straight beam into the final position due to its deformation. The four Euler-Rodrigues parameters must verify the relation:

$$
\boldsymbol{l}^{\mathrm{T}} \boldsymbol{l}=1 .
$$

Knowing the Euler-Rodrigues parameters, the rotation matrix from the initial configuration to the actual one, for each cross-section, has the form:

$$
\mathfrak{R}=\left[\begin{array}{lll}
2\left(l_{0}^{2}+l_{1}^{2}\right)-1 & 2\left(l_{1} l_{2}-l_{0} l_{3}\right) & 2\left(l_{1} l_{3}+l_{0} l_{2}\right) \\
2\left(l_{l} l_{2}+l_{0} l_{3}\right) & 2\left(l_{0}^{2}+l_{2}^{2}\right)-1 & 2\left(l_{2} l_{3}-l_{0} l_{1}\right) \\
2\left(l_{l} l_{3}-l_{0} l_{2}\right) & 2\left(l_{2} l_{3}+l_{0} l_{1}\right) & 2\left(l_{0}^{2}+l_{3}^{2}\right)-1
\end{array}\right] .
$$

To solve the problem, the total potential energy (1) has to be minimized and usually numerical methods are used, finite element method being the most effective. The classical large displacement finite element approach considers as nodal unknowns (nodal generalized coordinates) small increments of nodal displacements and rotations and this approach leads to an extremely timeconsuming incremental formulation.

In this work the unknowns are axial deformation $\varepsilon_{0}$ and the four EulerRodrigues parameters $\boldsymbol{l}$. They are grouped into the generalized coordinate vector:

$$
\boldsymbol{q}=\left(\begin{array}{lllll}
\varepsilon_{0} & l_{0} & l_{1} & l_{2} & l_{3}
\end{array}\right)^{T} .
$$


Curvature vector may be easily expressed in Euler-Rodrigues parameters as:

$$
\boldsymbol{\kappa}=\mathbf{G} \boldsymbol{l}^{\prime},
$$

where $l^{\prime}$ is the space derivative that is with respect to the curvilinear coordinates $s$. The $\mathbf{G}$ matrix has the form:

$$
\mathbf{G}=2\left[\begin{array}{cccc}
-l_{1} & l_{0} & l_{3} & -l_{2} \\
-l_{2} & -l_{3} & l_{0} & l_{1} \\
-l_{3} & l_{2} & -l_{1} & l_{0}
\end{array}\right]
$$

The equations are written in the actual reference frame of the current crosssection and therefore the spatial derivatives are performed with respect of actual curvilinear coordinate $S$ different of the curvilinear coordinate $s$ for the initial configuration. As the small strain hypothesis was considered, we may consider that the length of the beam keep its length during the deformation: $d S=\left(1+\varepsilon_{0}\right) d s \approx d s$. Thus the following symbolic expression may be written:

$$
\frac{\partial}{\partial S} \approx \frac{\partial}{\partial s} \text {. }
$$

In the local reference frame, virtual small rotation vector is expressed in EulerRodrigues parameters as:

$$
\delta^{L} \boldsymbol{\varphi}=\mathbf{G} \delta l
$$

and in the global reference frame:

$$
\delta \varphi=\Re \mathbf{G} \delta l .
$$

Finally the displacements vector $\boldsymbol{u}$ of the current point has the form:

$$
\boldsymbol{u}(s)=\boldsymbol{u}_{0}+\int_{0}^{s}\left(\Re \boldsymbol{n}\left(1+\varepsilon_{0}\right)-\boldsymbol{n}\right) d s=\boldsymbol{u}_{0}+\int_{0}^{s}\left(\left\{\begin{array}{l}
2\left(l_{0}^{2}+l_{1}^{2}\right)-1 \\
2\left(l_{1} l_{2}+l_{0} l_{3}\right) \\
2\left(l_{1} l_{3}-l_{0} l_{2}\right)
\end{array}\right\}\left(1+\varepsilon_{0}\right)-\left\{\begin{array}{l}
1 \\
0 \\
0
\end{array}\right\}\right) d s,
$$

where $\boldsymbol{n}$ is the unit vector perpendicular to cross-section. Now there are all the ingredients to solve numerically the problem using the variational formulation (1).

\section{Kinematic equations}

In the case of the beam in contact with the tube, the deformed shape of the beam is given by the equations:

$$
\left\{\begin{array}{l}
x=x(s) \\
y=R \cos \varphi(s) \\
z=R \sin \varphi(s)
\end{array}\right.
$$

The following relations exists between angles $\theta, \varphi$ (see figure 1) and the abscise $x$ (for small axial strain $\varepsilon_{0}$ ): 


$$
\begin{array}{llll}
d x=\sin \theta d s & \text { or } & \sin \theta=\frac{d x}{d s} \\
d \varphi=\frac{\cos \theta d s}{R} & \text { or } & \frac{d \varphi}{d s}=\frac{\cos \theta}{R}
\end{array}
$$

$R$ being the radius of the tube, figure 1 . Therefore the director parameters of an infinite small segment $\mathrm{d} s$ of the beam centre-line are:

$$
\frac{\mathrm{d} x}{\mathrm{~d} s}=\sin \theta, \quad \frac{\mathrm{d} y}{\mathrm{~d} s}=-R \cos \theta \sin \varphi, \quad \frac{\mathrm{d} z}{\mathrm{~d} s}=R \cos \theta \cos \varphi,
$$

where the angles $\theta$ and $\varphi$ result from figure 1.That must coincide to the first column of the matrix $\mathfrak{R}$ given by (7):

$$
\left\{\begin{array}{c}
l_{0}^{2}+l_{1}^{2}=\frac{1+\sin \theta}{2} \\
l_{1} l_{2}+l_{0} l_{3}=-\frac{\cos \theta \sin \varphi}{2} \\
l_{1} l_{3}-l_{0} l_{2}=\frac{\cos \theta \cos \varphi}{2}
\end{array} .\right.
$$

It is easy to show that the Euler-Rodrigues parameters satisfying equations (17), satisfy also equation (6).

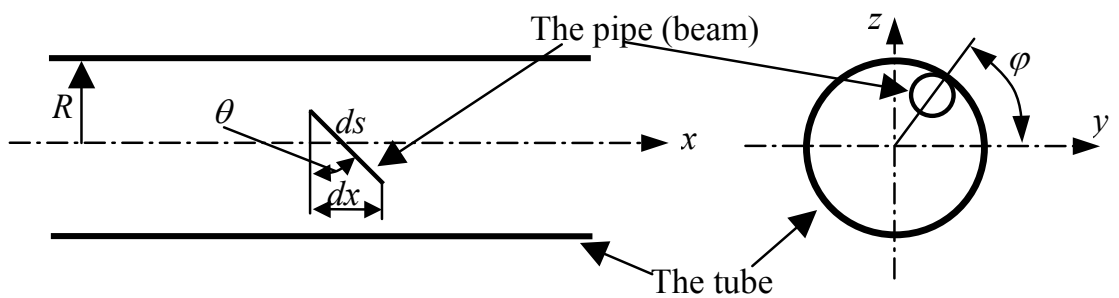

Figure 1: Definition of angles $\theta$ and $\varphi$.

The system resulting from (1) is nonlinear and the iterative Newton-Raphson method will be applied, that is at each iteration the system is solved in unknown increments. Equations (17) become in increments:

$$
\left[\begin{array}{cccccc}
l_{0} & l_{1} & l_{2} & l_{3} & 0 & 0 \\
l_{3} & l_{2} & l_{1} & l_{0} & \frac{1}{2} \cos \theta \cos \varphi & -\frac{1}{2} \sin \theta \sin \varphi \\
-l_{2} & l_{3} & -l_{0} & l_{1} & \frac{1}{2} \cos \theta \sin \varphi & \frac{1}{2} \sin \theta \cos \varphi
\end{array}\right]\left\{\begin{array}{l}
\delta l_{0} \\
\delta l_{1} \\
\delta l_{2} \\
\delta l_{3} \\
\delta \varphi \\
\delta \theta
\end{array}\right\}=\left\{\begin{array}{l}
0 \\
0 \\
0 \\
0 \\
0 \\
0
\end{array}\right\},
$$

to which the incremental form of the second equation (15) is added: 


$$
\delta \varphi=-\int_{0}^{s} \frac{\sin \theta \delta \theta}{R}\left(1+\varepsilon_{0}\right) \mathrm{d} s+\int_{0}^{s} \frac{\cos \theta}{R} \delta \varepsilon_{0} \mathrm{~d} s .
$$

Equations (18) and (19) represent the relations between the increments of EulerRodrigues parameters and the increments of the two angles $\theta$ and $\varphi$. There are 4 equations, linking 7 unknown functions, $\varepsilon_{0}, l_{0}, l_{1}, l_{2}, l_{3}, \theta, \varphi$, imposing the deformed beam to lie on the internal surface of a tube of radius $\mathrm{R}$. Therefore only 3 independent generalized coordinates were chosen:

$$
\hat{\boldsymbol{q}}=\left(\begin{array}{lll}
\varepsilon_{0} & l_{1} & \theta
\end{array}\right)^{\mathrm{T}} .
$$

\section{Friction forces}

Between the beam and the lateral surface of the tube there are reaction forces. Moment equilibrium equations for a slice of the beam, written in the local reference frame of each cross-section, is:

$$
\frac{\partial \boldsymbol{M}}{\partial s}+\boldsymbol{n} \times \boldsymbol{R}=\mathbf{0} \quad \text { or } \quad \mathbf{D} \frac{\partial(\boldsymbol{\kappa})}{\partial s}+\boldsymbol{\kappa} \times(\mathbf{D} \boldsymbol{\kappa})+\boldsymbol{n} \times \boldsymbol{R}=\mathbf{0} .
$$

After each iteration, the generalized coordinates $\hat{\boldsymbol{q}}$ are known and the EulerRodrigues parameters are known as well; that allow the curvatures $\boldsymbol{\kappa}$ to be found. The axial effort $N$ and the two shear efforts $Q_{2}$ and $Q_{3}$ (the components of the vector $\boldsymbol{R}$ ) are computed using equation (21). Finally, from the force equilibrium equation written in the cross section local reference frame:

$$
\frac{\partial \boldsymbol{R}}{\partial s}+\boldsymbol{\kappa} \times \boldsymbol{R}+\boldsymbol{p}=\mathbf{0}
$$

it results the contact pressure $\boldsymbol{p}$ between the beam and the tube. Distributed force $p$ will be represented in the cylindrical reference frame. Axes of the cylindrical reference frame are: $1-x$ direction of the global frame (see figure 1), 2- normal direction and 3 - tangent direction in the $y z$ global plane. The normal distributed force is $p_{2}$. The friction force cannot exceed the value $\mu p_{2}$, where $\mu$ is the friction coefficient. If the tangential force is smaller than $\mu p_{2}$, there is no relative motion between the points in contact belonging to the beam and to the tube. In any point the following condition must be respected:

$$
\sqrt{\tilde{p}_{1}^{2}+\tilde{p}_{3}^{2}} \leq \mu \widetilde{p}_{2}
$$

otherwise the "link" is broken and a relative motion is allowed. At each load step few iterations are needed in order to establish which among all nodes are moving and which are not because of the friction.

The tube is compressed by an axial force with the condition that the tube always lies on the surface of the tube. In the absence of the friction the method is not incremental and the solution may be find directly for the final load of the load. But in the presence of the friction the approach must be incremental, the result depending on the history of the force variation. 

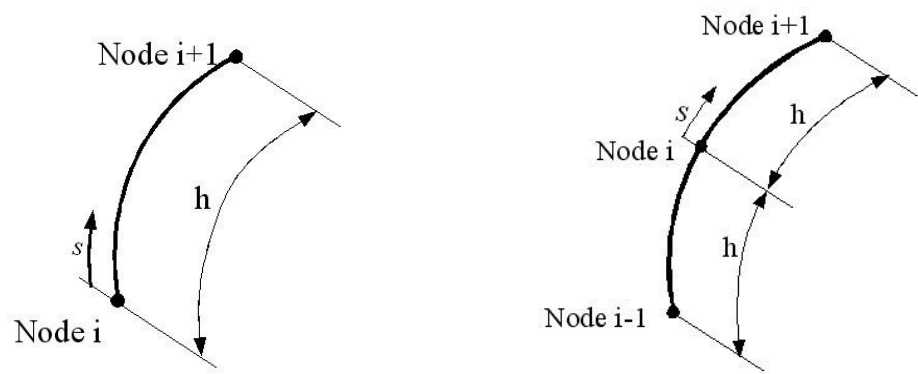

Figure 2: $\quad$ Two-node and three-node finite elements.

\section{Special finite element}

To solve the problem, finite element method was applied. Isoparametric twonode and three-node finite elements were used, figure 2. This finite element is described in detail in [15-17]. Each node has five degrees of freedom per node accordingly to (8). For the three-node finite element the shape function is:

$$
q_{\mathrm{k}}(s)=\left(\frac{s^{2}}{2 h^{2}}-\frac{s}{2 h}\right) q_{\mathrm{k}, \mathrm{i}-1}+\left(-\frac{s^{2}}{h^{2}}+1\right) q_{k, i}+\left(\frac{s^{2}}{2 h^{2}}+\frac{s}{2 h}\right) q_{k, i+1}, k=1 \ldots .5 .
$$

The beam is divided in several finite elements and the load is applied incrementally. Finally a non-linear differential system is obtained from functional (1) using a procedure similar to the standard one:

$$
E(q)=\mathbf{K}(q) q-Q=0,
$$

where $\mathbf{K}$ is the secant stiffness matrix that is function of $\boldsymbol{q}$, obtained by assembling elemental stiffness matrices; $\boldsymbol{q}$ is the vector of the generalized nodal unknown (8) for the whole structure and $\boldsymbol{Q}$ is the vector of nodal loads. System (25) includes the equation (6) for each node. It is interesting to note that all elements of secant stiffness matrix $\mathbf{K}$ are polynomials in Euler-Rodrigues $\boldsymbol{l}$ and strain $\varepsilon_{0}$.

To solve the non-linear system, the iterative Newton-Raphson method is applied. At each step, at each iteration, equations (18) and (19) are used in order to replace nodal degrees of freedom (8) with (20), to impose the beam to lie on the cylindrical surface of the tube. In the load step $i$ and for the $k$-th iteration it can be written:

$$
\hat{\mathbf{K}}_{\mathrm{t}}^{(k)} \delta \hat{\boldsymbol{q}}_{i}^{(k+1)}+\hat{\boldsymbol{E}}^{(k)}=0,
$$

where:

$$
\boldsymbol{q}=\mathbf{C} \hat{\boldsymbol{q}}, \mathbf{K}_{\mathbf{t}}=\mathbf{C}^{\mathrm{T}} \hat{\mathbf{K}}_{\mathbf{t}} \mathbf{C}, \mathbf{E}=\mathbf{C} \hat{\mathbf{E}},
$$

C being a square matrix provided by applying of equations (18) and (19) for all nodes of the discretization and $\delta \hat{\boldsymbol{q}}_{i}^{(k+1)}=\hat{\boldsymbol{q}}_{i}^{(k+1)}-\hat{\boldsymbol{q}}_{i}^{(k)} . \mathbf{K}_{\mathrm{t}}$ is the tangent stiffness modulus, in fact the Jacobean $\mathbf{J}$ : 


$$
\mathbf{J}=\mathbf{K}_{\mathrm{t}}=\frac{\partial \boldsymbol{E}}{\partial \boldsymbol{q}} .
$$

The approximate evaluation of the Jacobean may be used, although in the paper the exact computing were performed:

$$
\mathbf{K}_{\mathrm{t}} \approx \mathbf{K}+\frac{\partial \boldsymbol{Q}}{\partial \boldsymbol{q}} .
$$

Often an under-relaxation factor $\beta$ could improve the convergence:

$$
\hat{\boldsymbol{q}}_{i+1}^{(k+1)}=\hat{\boldsymbol{q}}_{i+1}^{(k)}+\beta \delta \hat{\boldsymbol{q}}_{i+1}^{(k+1)} .
$$

Angle $\theta\left[^{\circ}\right]$

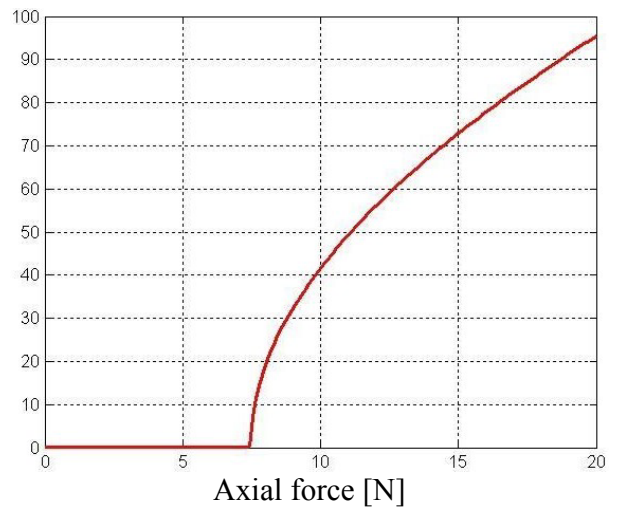

a) Rotation angle $\theta$ of the mid node versus axial force.

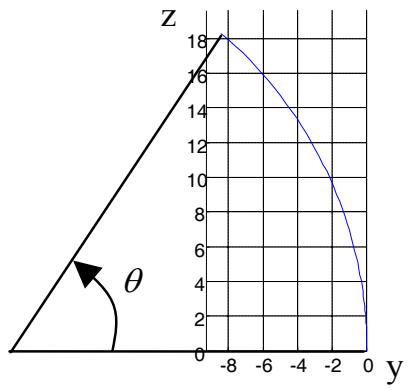

b) Beam (pipe) view along $\mathrm{x}$ axis, the beam axis.

Figure 3: Post-buckling diagram.

\section{Numerical example}

To illustrate the method a MATLAB program was elaborate and the following example was analyzed, $[6,7,13]$ : double hinged beam (pipe), length $\mathrm{L}=21340$ $\mathrm{mm}$, outer pipe diameter $\mathrm{D}=13.72 \mathrm{~mm}$, inner pipe diameter $\mathrm{d}=6.35 \mathrm{~mm}$, diameter of the tube $\mathrm{D}_{0}=48.26 \mathrm{~mm}$, Young modulus $\mathrm{E}=2.07 \mathrm{e} 5 \mathrm{MPa}$, Poisson coefficient $v=0.3,303$-node finite elements, 61 nodes. Figure 3 shows the dependence angle $\theta$ versus axial force, dependence found by the computer code. The Euler buckling force is $7.45 \mathrm{~N}$ (see figure 3a) and much higher force values were studied. Figure 4 presents the deformation configuration of the pipe under an axial force of $800 \mathrm{~N}$. One can see that the shape is not exactly a helix: the symmetry about the plane perpendicular on the beam, on the mid-node, has to be ensured. On the contrary, figure 5 shows the unsymmetrical deformed configuration of a clamped-free beam. 


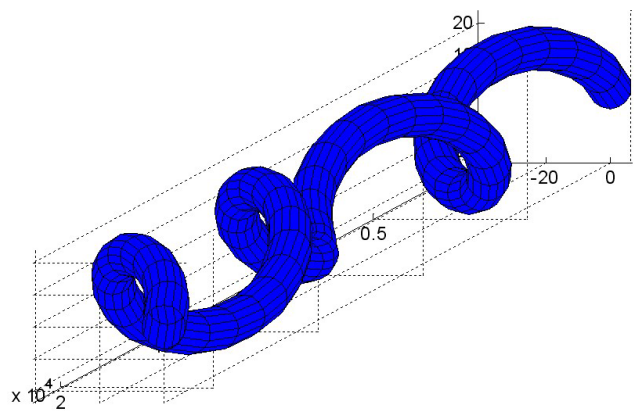

a) Isometric view (view direction: $[1 ; 0.006 ; 0.003]$ )

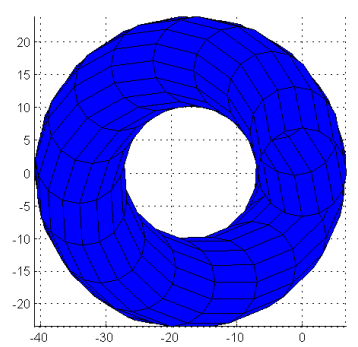

b) View along $x$ axis, the hole axis.

Figure 4: Post-buckling shape of a double hinged beam; compression force $800 \mathrm{~N}$.

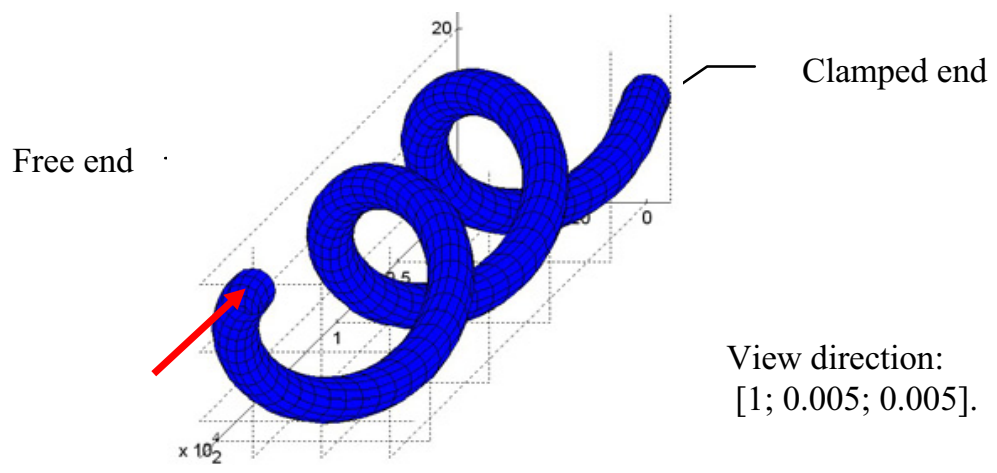

Figure 5: Post buckling configuration for a compressed clamped-free beam.

\section{Conclusions}

In the case of complex structural problems, numerical methods, especially those based on finite element method, may describe much more exactly the real phenomenon than analytical methods. This is also the case for long and slender beam compressed in a cylinder tube, the topic of the present work. A special finite element type, mainly conceived for geometrical non-linearity of beam systems, was proposed which proved to be fast convergent and exact. Equilibrium equations are exact and written on the deformed configuration of the beam: this is possible only because the generalized nodal coordinates are the elements of Euler-Rodrigues quaternion and the axial strain. In this way the boundary conditions can be described better and a significant influence of boundary conditions was found even for quite long beams (pipes).

\section{References}

[1] Sorenson, K.G., Post buckling behavior of a circular rod constrained within circular cylinder, PhD thesis, Rice University, Houston, Texas, 1984. 
[2] Chen, Yu-Che, Post buckling behavior of a circular rod constrained within an inclined hole, PhD thesis, Rice University, Houston, Texas, 1988.

[3] Miska, A \& Cunha, J.C., An analysis of helical Buckling of Tubulars Subjected to axial and torsional Loading in inclined wellbores, Society of Petroleum Engineers, SPE 29460, pp. 173-180, 1995.

[4] Tan, X.C. \& Forsman, B., Buckling of slender string in cylindrical tube under axial load: experiments and theoretical analysis, Experimental Mechanics, 35(1), pp. 55-60, 1995.

[5] Jang Wu \& Juvkam-Wold, H.C., Coiled tubing buckling implication in drilling and completing horizontal wells, SPE Drilling \& Completion, March, pp. 16-21, 1995.

[6] Miska, S., Qiu, W., Volk L. \& Cuhna, J.C., An improved analysis of axial force along coiled tubing in inclined, horizontal wellbores, Society of Petroleum Engineers, SPE 37056, pp. 207-214, 1996.

[7] Kuru, E., Martinez, A. \& Miska, S., The buckling behavior of pipes and its influence on the axial force transfer in directional wells, Society of Petroleum Engineers, SPE 53840, pp. 1-9, 1999.

[8] Qiu, W, Force transmission of coiled tubing in horizontal wells, Society of Petroleum Engineers, SPE 54584, pp. 1-7, 1999.

[9] Mitchell, R.F., Exact analytic solutions for pipe buckling in vertical and horizontal wells, SPE Journal, December, pp. 35-51, 2002.

[10] Mitchell, R.F., Lateral Buckling of pipes with connectors in horizontal wells, SPE Journal, June, pp. 41-52, 2003.

[11] Gao, De-Li, Gao, Bao-Kui, A method for calculating tubing behavior in HPHT wells, Journal of Petroleum Science \& Engineering, 41, pp. 183$188,2004$.

[12] Sun, C. \& Lukasiewicz, S., A new method on the buckling of a rod in tubing, Journal of Petroleum Science \& Engineering, 50, pp.78-82, 2006.

[13] Martinez, A., Miska, S., Kuru, E. \& Sorem, J., Experimental Evaluation of the Lateral Contact Force in Horizontal Wells, Journal of Energy Resources Technology, 122(3), pp. 123-128, 2000.

[14] Kuru, E. Martinez, A., Miska, S. \& Qiu, W., The buckling behavior of pipes and its influence on the axial force transfer in directional wells, Journal of Energy Resources Technology, 122(3), pp. 129-135, 2000.

[15] Barraco, A. \& Munteanu, M.Gh., A special finite element for static and dynamic study of mechanical systems under large motion", part 1, Revue européenne des éléments finis, Vol. 11(6), pp.773-790, 2002.

[16] Munteanu, M.Gh. \& Barraco, A., A special finite element for static and dynamic study of mechanical systems under large motion", part 2, Revue européenne des éléments finis, Vol. 11(6), pp.791-814, 2002.

[17] Barraco, A. \& Munteanu, M.Gh., Dynamic study of elastic beam systems under large motion, Proc. of European Congress on Computational Methods in Applied Sciences and Engineering, ECCOMAS, Finland, 24 28 July, published in extensor on CD, 2004. 